\title{
Efectos adversos sistémicos de los corticoides inhalatorios
}

Lipworth BJ. Systemic Adverse Effects of Inhaled Corticosteroid Therapy. A Systematic Review and Meta-analysis. Arch. Intern. Med. 1999;159:941-55.

\section{Objetivo}

Revisar los datos existentes sobre los efectos adversos sistémicos de los corticoides inhalatorios.

\section{Fuente y selección de datos}

El presente estudio es un meta-análisis. Se realizó una búsqueda utilizando MEDLINE, EMBASE y BIDS desde 1966 a 1998 incluyendo estudios que investigaran los efectos de los corticoides inhalatorios sobre la función suprarrenal, el crecimiento, el metabolismo óseo, la piel y el ojo. Se seleccionaron estudios farmacocinéticos y farmacodinámicos cuando éstos fueron relevantes. Se incluyeron estudios en voluntarios sanos y en niños y adultos asmáticos. Se realizó un meta-análisis de 27 estudios para evaluar el efecto de los distintos corticoides en la supresión adrenal.

\section{Resultados}

Tabla. Perfil farmacocinético de los corticoides inhalatorios

\begin{tabular}{lccccc}
\hline Corticoide & \multicolumn{2}{c}{$\begin{array}{c}\text { Liposolubilidad Primer paso } \\
\text { pulmonar }\end{array}$} & $\begin{array}{l}\text { Primer paso } \\
\text { hepático }\end{array}$ & $\begin{array}{c}\mathrm{T} 1 / 2 \\
\text { administración c/12 hs }\end{array}$ \\
\hline Fluticasona & +++ & - & +++ & $14,4 \mathrm{hs}$ & $\begin{array}{c}\text { Acumulación en la } \\
\text { admida }\end{array}$ \\
\hline Budesonida & - & +++ & $2,3 \mathrm{hs}$ & - \\
\hline Triamcinolona & ++ & - & +++ & $3,6 \mathrm{hs}$ & - \\
\hline Beclometasona & ++ & ++ & ++ & $6,5 \mathrm{hs}$ & - \\
\hline
\end{tabular}

Se observó una marcada supresión adrenal con los corticoides inhalatorios en dosis superiores a $1,5 \mathrm{mg} /$ día $(0,75 \mathrm{mg} /$ día para la fluticaso- na), aunque existió una considerable variabilidad interindividual. El meta-análisis demostró un mayor grado de supresión para la fluticasona comparada con la beclometasona, budesonida, y triamcinolona en dosis bioequivalentes, mientras que fue similar a la prednisolona sobre una relación de potencia de 10:1 mg. Los corticoides inhalados analizados mostraron una reducción significativa en la densidad ósea cuando fueron administrados en dosis superiores a las descriptas. Aunque estudios de crecimiento a mediano plazo con $400 \mathrm{microg} / \mathrm{d}$ de beclometasona, no se encontró evidencia suficiente sobre el efecto en la estatura final en la adultez. El uso de corticoides inhalatorios en altas dosis y a largo plazo aumenta el riesgo de catarata subcapsular y, en menor grado, de hipertensión ocular y glaucoma. El efecto adverso cutaneo más frecuente son los hematomas; estos se observan con altas dosis de corticoides y se correlacionan con el grado de supresión adrenal.

\section{Conclusiones}

Todos los corticoides inhalatorios poseen efectos adversos sistémicos dependientes de la dosis, aunque éstos son menores que los de los corticoides orales a dosis bioequivalentes. El presente meta-análisis muestra que la fluticasona posee una actividad sistémica mayor que los demás corticoides inhalatorios en dosis comparables, especialmente en dosis que superan los $0,8 \mathrm{mg} /$ día.

\section{COMENTARIO}

Los corticoides, tanto en su forma inhalatoria como sistémica, son altamente efectivos en el control del asma y la inflamación de la vía aérea. Las guías para el tratamiento del asma ${ }^{1-2}$ ubican a la terapia con corticoides inhalatorios en la primera línea del tratamiento antiinflamatorio a largo plazo. A la vez, existen claras recomendaciones tendientes a evitar los efectos adversos, como el uso de cámara espaciadora, la higienización bucal luego de la aplicación y el uso de la menor dosis posible. También se recomienda la adición de un agonista b2 al tratamiento junto con dosis bajas o medianas de corticoides en lugar de la utilización de altas dosis de estos últimos.

El presente estudio describe las características farmacológicas de los corticoides inhalatorios y analiza los efectos adversos sistémicos de los mismos. Como se advierte en el estudio, el meta-análisis muestra algunas limitaciones. Cuando se analizó la supresión adrenal, la fluticasona presentó una actividad sistémica mayor en dosis bioequivalentes. Sin embargo, la administración por vía inhalatoria dificulta la comparación, ya que es complejo calcular que parte de la dosis llega al sitio de acción y que parte se deglute o se absorbe en la mucosa yu- gat, las influencias de la higiene bucal y el uso de espaciadores. Asimismo los diferentes grados de obstrucción de la vía aérea en la población asmática alteran la disponibilidad de las drogas inhalatorias en el sitio de acción, y además la relación de potencia in vitro para distintos corticoides inhalatorios no puede trasladarse directamente al paciente.

También se encuentran dificultades al medir la influencia de el uso de corticoides en el crecimiento. Por un lado porque el asma es una causa de retraso en el mismo, y por el otro porque los métodos que miden crecimiento en el corto plazo no permiten predecir los efectos de los corticoides inhalados en el largo plazo.

No existen dudas que el uso de corticoides inhalatorios representa un avance en términos de riesgo-beneficio respecto de los corticoides orales en el tratamiento del asma como medicación a largo plazo. De todos modos, deben tenerse presentes las recomendaciones de utilizar las dosis mas bajas posibles, de combinarlos con medicaciones no esteroideas cuando sea necesario, y de pesquisar estigmas de efectos adversos sistémicos.

Dr. Daniel M. Casas

II Cátedra de Farmacología UBA

Dr. Sergio D. Zunino

Departamento de Fisiología UBA

Referencias

1. Global Initiative for Asthma Treatment. WHO. http \|www.ginasthma.com

2. National Asthma Education and Prevention Program. Expert Panel Report 2: Guidelines for the Diagnosis and Management of Asthma. Bethesda, Md: National Institutes of Health; 1997.

Publication 97-4051.

Study. Lancet 1999; 354: 471-76.

11. Newell M.L. Infant Feeding And Hiv 1 Transmission. Lancet 1999; 354: 442-443. 